\title{
Associations between the 2007 Medicare reimbursement reduction for bone mineral density testing and osteoporosis drug therapy patterns of female Medicare beneficiaries
}

\author{
This article was published in the following Dove Press journal: \\ Patient Preference and Adherence \\ 25 June 2014 \\ Number of times this article has been viewed
}

Sun Jung Kim ${ }^{1,2}$

Joo Hun Lee ${ }^{3}$

Sulgi Kim ${ }^{4}$

Shunichi Nakagawa ${ }^{5}$

Heather Bertelson ${ }^{6}$

Julia Lam $^{7}$

Ji Won Yoo ${ }^{6,7}$

'Department of Public Health, ${ }^{2}$ Institute of Health Services Research, Yonsei University College of Medicine, ${ }^{3}$ Department of Media and Communication, Hanyang University College of Social Sciences, Seoul, Republic of Korea;

${ }^{4}$ Department of Epidemiology and Biostatistics, Case Western Reserve University, Cleveland, OH, USA; ${ }^{5}$ Department of Medicine, College of Physicians and Surgeons, Columbia University, New York, NY, USA; ' ${ }^{6}$ epartment of Internal Medicine, Aurora Health Care, Milwaukee, WI, USA; ${ }^{7}$ Department of Medicine, University of Wisconsin School of Medicine and Public Health, Madison, WI, USA

Video abstract

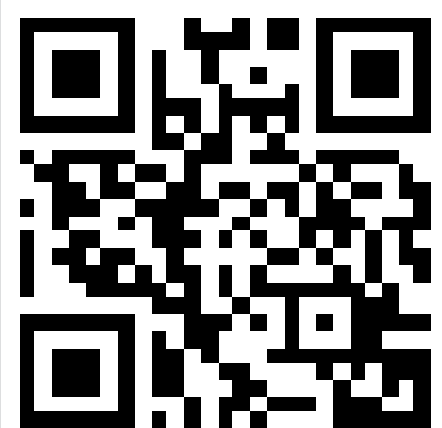

Point your SmartPhone at the code above. If you have a QR code reader the video abstract will appear. Or use: http://dvpr.es/lkJfCIL

Correspondence: Ji Won Yoo

Aurora Health Care, $1020 \mathrm{~N}$ 12th Street, Suite 30I, Milwaukee, WI, USA

Tel + I 4142197300

Fax +I 4142197632

Email yoo36@wisc.edu
Objective: To examine how drug therapy patterns for osteoporosis have changed after the Medicare Physician Fee Schedule (MPFS) reimbursement reduction in 2007, in relation to follow-up bone mineral density (BMD) testing status.

Methods: We used a retrospective temporal shift design to examine changes in drug therapy patterns before (Phase 1: January 1, 2005-December 31, 2006) and after (Phase 2: July 1, 2007June 30, 2009) the MPFS reimbursement reduction in 2007, Cleveland, OH, USA. Participants were osteoporotic older women in Phase $1(n=1,340)$ and Phase $2(n=1,437)$. The main outcomes were a) adherence, b) adjustment, c) occurrence of an extended gap, and d) restarting drug therapy after an extended gap. Follow-up BMD testing status by study phase and location were also analyzed.

Results: BMD testing rates at physicians' offices decreased from $64.5 \%$ in Phase 1 to $58.4 \%$ in Phase 2 ( $P=0.02$ ); however, testing rates in hospital outpatient settings increased (from $20.8 \%$ to $24.5 \%$ ). There were also decreases in drug therapy adjustment from $15.9 \%$ in Phase 1 to $11.6 \%$ in Phase 2 (odds ratio [OR]: $0.73 ; P<0.01$ ) and in restarting drug therapy after an extended gap (55.4\% in Phase 1 and $43.6 \%$ in Phase 2; OR: $0.76 ; P<0.01)$.

Conclusion: There were no changes in the overall rate of follow-up BMD testing. The rates of drug adjustments and restarting drug therapy after an extended gap did decrease. These decreases were more evident when follow-up BMD testing was not performed.

Keywords: accessibility of health services, drug therapy, osteoporosis, elderly women

\section{Introduction}

Osteoporosis is a common bone mineral disease responsible for 2 million fractures in the US yearly, particularly in older women. ${ }^{1}$ Its early detection and treatment can prevent fractures as well as new disabilities, and eventually, reduce health care costs. ${ }^{1}$ The current "gold standard" for measuring bone mineral density (BMD) is central dual-energy X-ray absorptiometry. ${ }^{1}$

Follow-up BMD testing for osteoporotic patients is covered entirely by Medicare every 2 years, or more often if necessary. ${ }^{1}$ The Medicare Physician Fee Schedule (MPFS) is the fee for physician services reimbursed by Medicare. However, the MPFS reimbursement rate for BMD testing in physicians' offices was reduced from US\$139 in 2006 to US\$82 from 2007-2008 and then further to US\$50 in 2013..$^{2,3}$ The rationale of the reimbursement reduction was to save Medicare an estimated US\$2.8 billion over 5 years. ${ }^{2}$ The MPFS rate reduction did not apply, however, to the reimbursement rate in hospital outpatient settings. ${ }^{2}$ 
There is growing concern regarding how the recent MPFS reimbursement reduction might affect osteoporosis treatments. ${ }^{4}$ This reduction could negatively affect monitoring and detection of osteoporosis as it may reduce BMD testing in physicians' offices through the introduction of barriers such as complicated intake procedures, reduced access, and increased travel expenses, according to The Lewin Group (Falls Church, VA, USA), an independent national health care and human services policy research consulting firm. ${ }^{4}$ Another recent study suggests that the MPFS reimbursement reduction appeared to be concurrent with reductions in clinicians' motivations to invest in and to educate the public about BMD testing, especially at lowvolume testing sites. ${ }^{5}$ However, these effects have yet to be thoroughly examined.

The annual rate of BMD testing in physicians' offices has been on the decline since the initial MPFS reduction. ${ }^{6}$ This is a concern, as follow-up BMD testing has been linked to adherence, which is crucial in the treatment of osteoporosis. Only approximately half of all osteoporotic patients adhere to drug therapy for at least 1 year. ${ }^{7,8}$ This low adherence is problematic, because fracture rates do not differ between patients who show $50 \%$ adherence to bisphosphonate (BP) therapy and those who do not receive any medication. ${ }^{8}$ Moreover, fractures and new disabilities may result from low adherence to drug therapy in osteoporotic patients. ${ }^{1,7,8}$ Osteoporotic patients have been found to be more likely to adhere to drug therapy if they undergo follow-up BMD testing; however, this study was limited to male veterans. ${ }^{9}$ Nevertheless, it seems probable that follow-up testing can promote adherence and thereby decrease the burden of osteoporosis.

Unfortunately, the optimal interval or frequency for follow-up BMD testing has not yet been determined. ${ }^{1,10}$ The ongoing controversy surrounding the efficacy of follow-up BMD testing for monitoring osteoporosis drug treatments has yet to be resolved. ${ }^{11-13}$ Previous findings have suggested that monitoring drug therapy through BMD testing in postmenopausal women in the first 3 years after the start of BP therapy is unnecessary and may be misleading. ${ }^{11,12}$ However, Watts et al countered that it was premature to conclude the efficacy of follow-up BMD testing for monitoring osteoporosis drug therapy based on the research that has been conducted thus far. ${ }^{13}$

Drug treatments are both dynamic and complex, especially in the management of chronic diseases. It is difficult to interpret drug therapy patterns based on a single outcome, such as adherence. Patients may need to have their drug treatments adjusted, or restarting drug therapy after experiencing a treatment gap. Therefore, the present study examines changes in drug therapy patterns, rather than adherence alone, after the initial MPFS reimbursement reduction. Specifically, we examined: 1) adherence, adjustment, occurrence of extended gaps, and restarting drug treatment after a gap and 2) how drug therapy patterns changed according to follow-up BMD testing status.

\section{Methods}

\section{Study design and data collection}

The study site was the Cleveland Clinic Health System, the predominant non-profit health care system in the metropolitan Cleveland area, OH, USA. This study was composed of two phases: Phase 1, before MPFS reduction, and Phase 2, after MPFS reduction. Each phase consisted of a 3-month look-back period (Phase 1: October 1, 2004December 31, 2004; Phase 2: April 1, 2007-June 30, 2007) and a subsequent 24-month observation period (Phase 1: January 1, 2005-December 31, 2006; Phase 2: July 1, $2007-$ June 30, 2009). Because the period of time covered entirely by Medicare for the BMD testing is 2 years, and the majority of previous studies conducted by osteoporosis-related professional societies adopted a 2-year time frame, we likewise determined 24 months to be the best observation period for both study phases. ${ }^{1,14}$ Inclusion criteria were: a) being a female Medicare beneficiary and b) having begun new osteoporosis drug therapy (not restarting drug therapy) during the look-back period with one of the US Food and Drug Administration (FDA)-approved medications (BP, selective estrogen receptor modulator [SERM], calcitonin, and teriparatide). BP included either brand or generic alendronate, ibandronate, risedronate, and zoledronate. SERM included raloxifene. Exclusion criteria were: being treated with a) denosumab or b) hormone therapy that had not been approved for osteoporosis treatment by the FDA during the study period. ${ }^{1}$

The health system's integrated electronic medical records (EMRs), were connected to diagnostic testing facilities and retail pharmacies in the vicinity, and thus, were considered feasible research tools. ${ }^{14}$ There was no patient who was assigned to both Phases 1 and 2 simultaneously. We confirmed this using medical record number and name in the EMR. Researchers were able to access information regarding osteoporosis treatments and BMD testing statuses through the EMRs. We accessed the EMRs of other health systems in their geographical locations. BMD testing was performed at either a physician's office (defined as an independent 
diagnostic facility located outside a hospital) or hospital outpatient settings (encompassing diagnostic facilities and services located within a hospital).

Three hundred and thirty-four study participants were withdrawn from the study due to insufficient datasets. Two hundred and twenty-seven study participants either died or were withdrawn due to serious health conditions (eg, glomerular filtration rate [GFR] $\leq 30 \mathrm{~mL} /$ minute $/ 1.73 \mathrm{~m}^{2}$ ) that contraindicated any osteoporosis drug therapy. Data on a total of 2,777 participants (1,340 in Phase 1 and 1,437 in Phase 2) were eventually analyzed. The study was approved by the institutional review board of the Cleveland Clinic Health System. Informed consent from patients was waived due to the use of secondary data.

\section{Main outcomes}

As the term "adherence" is synonymous with "medication compliance", according to the International Society for Pharmacoeconomics and Outcomes Research, we used the term in our study to mean likewise. ${ }^{15}$ The main outcomes were: a) adherence, that is, whether participants adhered to osteoporosis drug therapy during the 24-month follow-up period; b) adjustment, or whether the osteoporosis drug therapy class, name, strength, or frequency was adjusted without any extended gaps in treatment; $c$ ) the occurrence of an extended gap; and d) restarting drug therapy after an extended gap. Because an adherence rate of at least $80 \%$ was regarded as the minimum threshold for optimal efficacy in preventing fractures, we used a minimum of $80 \%$ of the days covered by drug therapy as an indicator of adherence. ${ }^{15,16}$ Adherence was measured using the medication possession ratio, which was defined as the number of actual drug fills divided by the number of potential fills. ${ }^{16}$ When clinicians discontinued drug therapy, the reason and the months of possible fills to that date were indicated. If participants discontinued drug therapy without endorsement from their clinicians and/or the drug had not been refilled for 6 consecutive months or longer, an extended gap was considered to have occurred.

\section{Covariates}

The covariates were age, race, education, smoking and/or drinking, supplementary private health insurance coverage, prior low trauma fracture at age 50 years or older, prolonged glucocorticoid use, acute hospitalization, a history of rheumatoid arthritis or immunosuppressant use, cognitive impairment, follow-up BMD testing, and drug profile (ie, osteoporosis treatment drugs captured during look-back periods).
Smoking and/or drinking was defined as currently smoking and/or having $3+$ drinks/day. ${ }^{1}$ Both smoking and drinking have been reported to be predictors of non-adherence to osteoporosis drug therapy. ${ }^{1}$ Meanwhile, supplementary private health insurance included Medicare Part D, which assists with additional out-of-pocket health care costs, and managed health care plans. Having supplementary private health insurance has been demonstrated to facilitate compliance with health maintenance programs, including for osteoporosis. ${ }^{14}$ Prolonged glucocorticoid use was defined as any use of oral prednisone equivalent to $5 \mathrm{mg}$ /day for more than 3 months. ${ }^{1}$ Acute hospitalization was defined as any hospitalization in the past 12 months that may interrupt osteoporosis pharmacotherapy maintenance through medical conditions that contraindicated osteoporosis drug therapy (eg, acute renal failure) or the unintentional gaps (pauses) in drug treatments it could create during care transitions (eg, being discharged from the hospital into the community). ${ }^{17}$ Osteoporosis drug therapy has been reported to be inversely associated with cognitive impairment. ${ }^{18,19}$ In our study, BMD testing was limited to the axial/central skeleton (spine and hip). Although previous studies ${ }^{11,12}$ on BMD testing have used other forms of bone mass measurement (eg, peripheral skeleton and quantitative computed tomography), these were less commonly performed and did not directly reflect the guidelines for monitoring osteoporosis drug therapy. Therefore, these forms were not included in the present study.

\section{Statistical analyses}

Bivariate comparisons of participant characteristics between study phases were examined using chi-square tests for categorical data and either Student's $t$-tests or Wilcoxon ranksum tests for continuous data. Follow-up BMD testing was stratified by study phases, locations, and outcomes. Univariate and multivariate logistic regressions of four outcomes were performed. These regressions were further stratified by follow-up BMD testing status. In multivariate regressions, the covariates were included as a simultaneous entry. Forward and backward stepwise selections were employed to ensure that the method of selecting variables had no impact on the regression models. Univariate and multivariate odds ratios (OR) and corresponding 95\% confidence intervals (CI) were derived, where $\mathrm{OR}>1$ indicated that the probability in Phase 2 was higher than that in Phase 1. Calculation of the variance inflation factor indicated no significant multicollinearity among the covariates. The reliability of the logistic regression models was assessed with Hosmer-Lemeshow Goodness-of-Fit tests: none of the fit criteria were violated. 
Table I Baseline characteristics by study phase

\begin{tabular}{|c|c|c|c|}
\hline & Phase I, n=I,340 & Phase 2, n=I,437 & $P$-value ${ }^{a}$ \\
\hline Age (years), mean ( \pm standard deviation) & $78.6(71.2-84.0)$ & $77.9(70.3-81.3)$ & 0.22 \\
\hline Non-whites, \% (n) & $30.5 \%(408)$ & $31.9 \%(458)$ & 0.47 \\
\hline Education (years), mean ( \pm standard deviation) & $8.1(4.9-9.8)$ & $7.9(5.0-10.3)$ & 0.24 \\
\hline Smoking and/or drinking, \% (n) & $14.7 \%(197)$ & $13.0 \%(187)$ & 0.33 \\
\hline Supplementary private health insurance, \% (n) & $38.2 \%(5 \mid 2)$ & $38.9 \%(560)$ & 0.57 \\
\hline Prior low trauma fracture at age 50 years or older, \% (n) & $13.4 \%(179)$ & $13.9 \%(201)$ & 0.68 \\
\hline Prolonged glucocorticoid use, \% (n) & $5.9 \%(80)$ & $6.6 \%(95)$ & 0.29 \\
\hline Acute hospitalization, \% (n) & $50.4 \%(675)$ & $49.9 \%(7 \mid 8)$ & 0.53 \\
\hline History of rheumatoid arthritis or any immunosuppressive drug use, \% (n) & $8.5 \%(114)$ & $7.00 \%(100)$ & 0.11 \\
\hline Cognitive impairment, \% (n) & $23.5 \%(315)$ & $26.0 \%(374)$ & 0.18 \\
\hline \multicolumn{4}{|l|}{ Osteoporosis drug therapy profile, \% (n) } \\
\hline Bisphosphonate & $81.6 \%(1,094)$ & $77.6 \%(1,116)$ & 0.09 \\
\hline Selective estrogen receptor modulator & $9.3 \%(125)$ & $12.6 \%(|8|)$ & \\
\hline Calcitonin & $4.4 \%(59)$ & $4.1 \%(59)$ & \\
\hline Teriparatide & $4.6 \%(62)$ & $5.6 \%(8 I)$ & \\
\hline
\end{tabular}

Note: ${ }^{\text {a }}$-values are derived from bivariate covariate comparisons by study phases.

All data procedures and analyses were performed using the SAS statistical software package (v9.3; SAS Institute, Inc., Cary, NC, USA), with a threshold of 0.05 for statistical significance (two-sided).

\section{Results}

\section{Participant characteristics}

Table 1 presents participant characteristics by study phases, which did not differ significantly. Phase 2 participants used more non-BP class drugs in Phase 2, with a statistically marginal difference $(P=0.09)$.

\section{Follow-up BMD testing stratified by study phases, locations, and outcomes}

Table 2 presents data on follow-up BMD testing stratified by study phases, locations, and outcomes. There were no significant differences in the percentages of follow-up BMD testing between the two study phases (Phase 1: 40.7\%; Phase 2: $39.3 \% ; P=0.37)$. However, there was a shift in BMD testing locations between the two study phases $(P=0.02)$ : the occurrence of BMD testing in physicians' offices decreased from $64.5 \%$ to $58.4 \%$, while BMD testing done in hospital outpatient settings increased from $20.8 \%$ to $24.5 \%$. There was no significant difference in the percentages of follow-up BMD testing when comparing certain outcomes between the two phases, except that there was a decrease in follow-up BMD testing from $48.5 \%$ in Phase 1 to $34.0 \%$ in Phase $2(P<0.01)$ among those restarting therapy after an extended gap.

\section{Drug therapy patterns stratified by study phases and follow-up BMD testing status}

Table 3 presents our multivariate logistic regression results on: a) adherence, b) adjustment, c) occurrence of an extended gap, and d) restarting drug therapy after an extended gap. After the MPFS reimbursement reduction for BMD testing, the likelihood of drug adjustment decreased significantly from Phase $1(15.9 \%)$ to Phase 2 (11.6\%; OR: 0.73, 95\% CI: 0.45$0.98 ; P<0.01)$. The likelihood of restarting drug therapy after an extended gap also decreased from Phase 1 (55.4\%)

Table 2 Follow-up bone mineral density testing stratified by study phase, location, and outcomes

\begin{tabular}{|c|c|c|c|}
\hline$\%(n)$ & Phase $I, n=I, 340$ & Phase 2, n=l,437 & $P$-value \\
\hline Follow-up bone mineral density testing, $\mathrm{n}=\mathrm{I}, \mathrm{I} \mid \mathrm{O}$ & $40.7 \%(547)$ & $39.3 \%(563)$ & 0.37 \\
\hline Locations & $\mathrm{n}=547$ & $\mathrm{n}=563$ & \\
\hline A) Physician office settings, $n=688$ & $64.5 \%(353)$ & $58.4 \%(329)$ & 0.02 \\
\hline B) Hospital outpatient settings, $n=245$ & $20.8 \%(114)$ & $24.5 \%(138)$ & \\
\hline Both $A$ and $B, n=177$ & $14.6 \%(80)$ & $17.1 \%(96)$ & \\
\hline \multicolumn{4}{|l|}{ Drug therapy patterns } \\
\hline Adherence to drug therapy & $33.5 \%(239 / 7 \mid 3)$ & $34.1 \%(250 / 747)$ & 0.74 \\
\hline Adjusting drug therapy & $40.2 \%(86 / 214)$ & $39.8 \%(67 / 168)$ & 0.59 \\
\hline Occurrence of extended treatment gap & $34.1 \%(124 / 363)$ & $36.1 \%(160 / 443)$ & 0.43 \\
\hline Restarting drug therapy after extended gap & $48.5 \%(98 / 202)$ & $34.0 \%(66 / 194)$ & $<0.01$ \\
\hline
\end{tabular}


Table 3 Drug therapy patterns stratified by study phases and follow-up bone mineral density testing status

\begin{tabular}{|c|c|c|c|c|}
\hline \multirow[t]{2}{*}{ Outcomes } & Phase I, $n=I, 340$ & Phase 2, n=I,437 & \multirow[t]{2}{*}{$\mathrm{OR}^{\mathrm{a}}(95 \% \mathrm{Cl})$} & \multirow[t]{2}{*}{ P-value } \\
\hline & \multicolumn{2}{|l|}{$\%(n)$} & & \\
\hline a) Adherence to drug therapy & $53.2 \%(7 / 3 / 1,340)$ & $51.98 \%(747 / I, 437)$ & $0.96(0.68-1.35)$ & 0.48 \\
\hline Follow-up BMD & $55.8 \%(239 / 428)$ & $51.4 \%(250 / 486)$ & $0.92(0.65-1.28)$ & 0.31 \\
\hline No follow-up BMD & $52.0 \%(474 / 9 \mid 2)$ & $52.2 \%(497 / 95 I)$ & $1.04(0.59-1.57)$ & 0.76 \\
\hline \multirow{3}{*}{$\begin{array}{l}\text { b) Adjusting drug therapy } \\
\text { Follow-up BMD } \\
\text { No follow-up BMD }\end{array}$} & $15.9 \%(2|4 /|, 340)$ & II.6\% (I68/I,437) & $0.73(0.45-0.98)$ & $<0.01$ \\
\hline & $20.1 \%(86 / 521)$ & $17.2 \%(67 / 389)$ & $0.88(0.49-1.32)$ & 0.15 \\
\hline & $13.7 \%(\mid 13 / 819)$ & $7.9 \%(83 / I, 048)$ & $0.57(0.22-0.95)$ & $<0.01$ \\
\hline c) Occurrence of extended treatment gap & $27.1 \%(363 / 1,340)$ & $30.8 \%(443 / 1,437)$ & I.I3 (0.77-I.49) & 0.27 \\
\hline Follow-up BMD & $24.5 \%(124 / 604)$ & $27.0 \%(160 / 593)$ & I. $12(0.72-1.74)$ & 0.22 \\
\hline No follow-up BMD & $29.2 \%(215 / 736)$ & $31.9 \%(270 / 844)$ & $1.10(0.66-1.86)$ & 0.49 \\
\hline \multirow{3}{*}{$\begin{array}{l}\text { d) Restarting drug therapy after extended gap } \\
\text { Follow-up BMD } \\
\text { No follow-up BMD }\end{array}$} & $55.4 \%(202 / 375)$ & $43.6 \%(194 / 431)$ & $0.76(0.47-0.98)$ & $<0.01$ \\
\hline & $52.3 \%(98 / / 47)$ & $56.4 \%(66 / 118)$ & I.07 (0.67-I.57) & 0.55 \\
\hline & $54.8 \%(125 / 228)$ & $32.2 \%(10|/ 3| 3)$ & $0.58(0.13-0.93)$ & $<0.01$ \\
\hline
\end{tabular}

Notes: a $O$ Rere derived from multivariate logistic regressions after adjusting for covariates. OR $>$ I indicated that the probability in Phase 2 was higher than that in Phase I; bno follow-up BMD indicated that follow-up BMD testing was not taken during the observational period.

Abbreviations: $\mathrm{BMD}$, bone mineral density; $\mathrm{Cl}$, confidence interval; $\mathrm{OR}$, odds ratio.

to Phase 2 (43.6\%; OR: $0.76,95 \%$ CI: $0.47-0.98 ; P<0.01)$. When follow-up BMD testing was performed, the observed differences between the two phases in drug adjustment and restarting drug therapy after an extended gap became non-significant. However, when follow-up BMD testing was not performed, the differences between the two phases widened. Drug adjustment decreased by a larger margin from Phase 1 (13.7\%) to Phase 2 (7.9\%; OR: 0.57, 95\% CI: 0.22 $0.95 ; P<0.01)$, and restarting drug therapy after an extended gap decreased from Phase $1(54.8 \%)$ to Phase 2 (32.2\%; OR: $0.58,95 \%$ CI: $0.13-0.93 ; P<0.01)$.

There were no significant differences between the two phases in terms of adherence to drug therapy (Phase 1: 53.2\%; Phase 2: $51.9 \% ; P=0.48)$ and occurrence of an extended treatment gap (Phase 1: 27.1\%; Phase 2: 30.8\%; $P=0.27$ ), even after stratification by follow-up BMD testing status. The results of the univariate analyses between the phases and outcomes were similar; therefore, these were not presented in the tables.

\section{Discussion}

To the best of our knowledge, this is the first study to explore how osteoporosis drug treatment patterns changed according to follow-up BMD testing status after the MPFS reimbursement reduction. In particular, we observed decreases in drug adjustment and restarting treatment after an extended gap. When follow-up BMD testing was performed, these became non-significant. However, in the absence of such testing, the difference between the two study phases widened.

These findings are important because evidence on this topic is currently mixed.
One interesting question that arises from these results follows: Why did certain changes in drug therapy patterns occur after the MPFS reimbursement reduction (eg, frequency of treatment adjustment and restarting drug therapy after an extended gap) as opposed to others (eg, adherence and occurrence of an extended treatment gap)?

It could be that patients going through a drug adjustment and restarting after an extended gap were more vulnerable to follow-up BMD testing than others. Follow-up BMD testing may not determine all occasions of drug treatment patterns in contrast to the previous studies. ${ }^{4,9}$

These observations may be explained by the dynamics of clinicians' prescription patterns. The annual rate of treatment adjustments for osteoporosis has previously been reported at approximately $10 \% .{ }^{20}$ Before clinicians decide to adjust or restart osteoporosis drug therapy, they usually prefer to conduct BMD testing and evaluate current treatments. ${ }^{10}$ Although the method of treatment might not be directly determined by the test results, BMD follow-up testing may provide clinicians, as well as patients, with the assurance they seek in steering their osteoporosis care plans. Therefore, the reduction in the MPFS reimbursement rate would discourage clinicians from using BMD tests more frequently in evaluating patients' current osteoporosis treatment plans. This would likely result in reduced osteoporosis care plan changes as shown in this study.

As described in previous reports, a shift of BMD testing location from physicians' offices to hospital outpatient settings was also observed in this study. This shift likely reflects decreased reimbursement in non-hospital employed physicians. ${ }^{3,21}$ 
Although Medicare provides reimbursement for BMD testing entirely every 2 years, these assessments have been underutilized. According to published reports using national data a decade earlier, the osteoporosis screening rates using BMD testing for Medicare females were less than 20\% and $22.9 \%$ in a 2- and 3-year time period, respectively. ${ }^{22,23} \mathrm{The}$ prevalence of BMD testing for follow-up purposes has rarely been examined, although a recent study of testing conducted since patients were first diagnosed with osteoporosis presented a figure of $38 \%$ during a 5 -year period. ${ }^{24}$ The relatively high rate of follow-up BMD testing in that study could have been associated with a recent quality of care project initiated at the health system level that promoted such testing in osteoporotic health care. ${ }^{25}$

Our findings on osteoporosis drug profile patterns were similar to those of previous studies. ${ }^{26} \mathrm{We}$ found that osteoporotic older women used non-BP more often than that reported previously. ${ }^{26}$ Our study participants were recruited relatively recently and used newer non-BP medications. In fact, more than $80 \%$ of Phase 1 participants used BPs. Phase 2 participants used more non-BP class drugs in Phase 2, though this difference was not statistically significant. This may be attributed to the fact that the number of non-BP users among osteoporotic drug treatment would likely increase further if future analyses included more recent data.

Our findings have practical, health care policy, and future research implications, even though we did not directly examine adverse outcomes of undertreatment (eg, BMD changes or fracture rates). The undertreatment of osteoporosis is relatively common; approximately two-thirds of women aged 55 years or older did not receive osteoporosis treatment within 1 year of diagnosis. ${ }^{26}$ Missing opportunities for timely drug adjustments or restarting treatments would raise red flags in the management and treatment of osteoporosis. Frequent, timely BMD testing could therefore improve the quality and outcomes of osteoporosis treatment through early detection of least significant change and minimization of extended gaps. ${ }^{9,13}$ Previous reports on the limited value of repeat BMD testing included healthy and untreated older females - a different population than reviewed in this study. ${ }^{27,28}$

The main strength of this study lies in its examination of the effects of the MPFS reimbursement reduction on both the BMD testing rate as well as osteoporosis drug therapy patterns. Further research is warranted to determine how the reduction would be associated with more direct osteoporosis outcomes such as BMD changes and fracture risks. The present study did have several limitations. First, our data analyses did not cover osteoporosis drug therapy patterns prescribed by clinicians that were not captured through the EMRs, and we could not quantify these cases. Location information on BMD testing was limited to hospital outpatient and physician office settings. More detailed information such as geographic site and test site volume was not available. For this reason, we acknowledge potential selection bias in our study. Second, our data (years 2005-2009) were premature and might not have reflected more recent BMD testing and drug therapy patterns during or after 2010. Indeed, a sharp drop in BMD testing rate in 2010 is expected because of further MPFS reimbursement reductions from US\$72 in 2009 to US\$50 in 2013. ${ }^{3}$ Third, we did not analyze clinicians' characteristics, which may have influenced follow-up BMD testing among osteoporotic patients. Clinician characteristics have been linked to the patterns in which BMD testing is ordered (eg, a greater number of BMD testing being ordered by female clinicians or in academic care settings). ${ }^{17}$ Finally, our findings cannot entirely reflect secular trends in the public awareness of the rare but important adverse effects of BPs (eg, atypical femoral fractures and jaw osteonecrosis) or the FDA's extension of the indications for SERM (raloxifene) for osteoporotic female breast cancer patients. ${ }^{29}$

\section{Conclusion}

Overall utilization of follow-up BMD testing did not change after the MPFS reimbursement reduction in 2007. Drug adjustment and restarting drug treatment did decrease, and this drop was more apparent when follow-up BMD testing was not conducted.

\section{Acknowledgments}

All authors confirm that all patient/personal identifiers have been removed or disguised so the patient/person(s) described are not identifiable and cannot be identified through the details of the story. This manuscript has not been under review or has not been published elsewhere. The authors thank Ms Susan Garcia, who helped prepare the manuscript, and Ms Brenda Fay for the literature search.

\section{Disclosure}

This study is supported by the American Geriatrics Society (Junior Researcher Seed Grant) and the Korean Ministry of Education, Science, and Technology (Excellence of Quality of Care Project). The funding sources had no role in the design and conduct of the study; collection, management, analysis, and interpretation of the data; and preparation, review, or approval of the manuscript. The authors report no conflicts of interest in this work. 


\section{References}

1. National Osteoporosis Foundation. 2013 Clinician's guide to prevention and treatment of osteoporosis. Washington, DC: National Osteoporosis Foundation; 2013. Available from: http://nof.org/hcp/clinicians-guide. Accessed March 9, 2014.

2. Federal Deficit Reduction Act. Proposed adjustments for payments to imaging services. Public Law Number 109-171; section 5102. Washington, DC: Center for Medicare and Medicaid Services; 2006.

3. Centers for Medicare and Medicaid Services [webpage on the Internet]. 2013 Coding and Reimbursement Guide: Bone densitometry at office and non-hospital imaging center. Medicare Physician Fee Schedule Look-up file. Baltimore, MD: Centers for Medicare and Medicaid Services; 2013. Available from: http://www.cms.gov/apps/physicianfee-schedule/license-agreement.aspx. Accessed March 9, 2014.

4. The Lewin Group [webpage on the Internet]. Assessing the costs of performing DXA services in the office-based setting. Falls Church, VA The Lewin Group; 2007. Available at: www.lewin.com/publications/ publication/114. Accessed March 9, 2014.

5. Hayes BL, Curtis JR, Laster A, et al. Osteoporosis care in the United States after declines in reimbursements for DXA. J Clin Densitom. 2010;13(4):3523-3560.

6. King AB, Fiorentino DM. Medicare payment cuts for osteoporosis testing reduced use despite tests' benefit in reducing fractures. Health Aff (Millwood). 2011;30(12):2362-2670.

7. Solomon DH, Avron J, Katz JN, et al. Compliance with osteoporosis medications. Arch Intern Med. 2005;165(20):2414-2419.

8. Siris ES, Harris ST, Rosen CJ, et al. Adherence to bisphosphonate therapy and fracture rates in osteoporotic women: relationship to vertebral and nonvertebral fractures from 2 US claims databases. Mayo Clin Proc. 2006;81(8):1013-1022.

9. Hansen KE, Swenson ED, Baltz B, Schuna AA, Jones AN, Elliott ME. Adherence to alendronate in male veterans. Osteoporos Int. 2008; 19(3):349-356.

10. Compston J. Monitoring osteoporosis treatment. Best Pract Res Clin Rheumatol. 2009;23(6):781-288.

11. Bell KJ, Hayen A, Macaskill P, et al. Value of routine monitoring of bone mineral density after starting bisphosphonate treatment: secondary analysis of trial data. BMJ. 2009;338:b2266.

12. Cummings SR, Palermo L, Browner W, et al. Monitoring osteoporosis therapy with bone densitometry: misleading changes and regression to the mean. Fracture Intervention Trial Research Group. JAMA. 2000;283(10):1318-1321.

13. Watts NB, Lewiecki EM, Bonnick SL, et al. Clinical value of monitoring BMD in patients treated with bisphosphonates for osteoporosis. J Bone Miner Res. 2009;24(10):1643-1646.

14. Yoo JW, Nakagawa S, Kim S. Effects of reimbursement reductions on bone mineral density testing for female Medicare beneficiaries. $J$ Womens Health (Larchmt). 2012;21(11):1144-1148.
15. Cramer JA, Roy A, Burrell A, et al. Medication compliance and persistence: terminology and definitions. Value Health. 2008;11(1):44-47.

16. Cadarette SM, Burden AM. Measuring and improving adherence to osteoporosis pharmacotherapy. Curr Opin Rheumatol. 2010;22(4):397-403.

17. Davisson L, Warden M, Manivannan S, et al. Osteoporosis screening: factors associated with bone mineral density testing of older women. J Womens Health (Larchmt). 2009;18(7):989-995.

18. Jennings LA, Auerbach AD, Maselli J, Pekow PS, Lindenauer PK, Lee SJ. Missed opportunities for osteoporosis treatment in patients hospitalized for hip fracture. J Am Geriatr Soc. 2010;58(4):650-657.

19. Colón-Emeric C, Lyles KW, Levine DA, et al. Prevalence and predictors of osteoporosis treatment in nursing home residents with known osteoporosis or recent fracture. Osteoporos Int. 2007;18(4):553-559.

20. Brookhart MA, Avorn J, Katz JN, et al. Gaps in treatment among users of osteoporosis medications: the dynamics of noncompliance. Am J Med. 2007;120(3):251-256.

21. Zhang J, Delzell E, Zhao H, et al. Central DXA utilization shifts from office-based to hospital-based settings among medicare beneficiaries in the wake of reimbursement changes. $J$ Bone Miner Res. 2012;27(4):858-864.

22. Patton E, Fischer H. Screening for osteoporosis in postmenopausal women: adherence to the 2002 USPSTF guidelines. Presented at: the Sixth International Symposium on Osteoporosis: Current Status, Future Directions; April 6, 2005; Washington, DC.

23. Neuner JM, Binkley N, Sparapani RA, Laud PW, Nattinger AB. Bone density testing in older women and its association with patient age. $J$ Am Geriatr. 2006;54(3):485-489.

24. McAdam-Marx C, Unni S, Ye X, Nelson S, Nickman NA. Effect of Medicare reimbursement reduction for imaging services on osteoporosis screening rates. J Am Geriatr Soc. 2012;60(3):511-516.

25. Olenginski TP, Antohe JL, Sunderlin E, Harrington TM. Appraising osteoporosis care gaps. Rheumatol Int. 2012;32(11):3619-3624.

26. Siris ES, Modi A, Tang J, Gandhi S, Sen S. Substantial undertreatment among women diagnosed with osteoporosis in a US managed-care population: a retrospective analysis. Curr Med Res Opin. 2014;30(1):123-130.

27. Berry SD, Samelson EJ, Pencina MJ, et al. Repeat bone mineral density screening and prediction of hip and major osteoporotic fracture. JAMA 2013;310(12):1256-1262.

28. Hillier TA, Stone KL, Bauer DC, et al. Evaluating the value of repeat bone mineral density measurement and prediction of fractures in older women: the study of osteoporotic fractures. Arch Intern Med. 2007;167(2):155-160

29. Diab DL, Watts NB. Bisphosphonates in the treatment of osteoporosis. Endocrinol Metab Clin North Am. 2012;41(3):487-506.
Patient Preference and Adherence

\section{Publish your work in this journal}

Patient Preference and Adherence is an international, peer-reviewed, open access journal that focusing on the growing importance of patient preference and adherence throughout the therapeutic continuum. Patient satisfaction, acceptability, quality of life, compliance, persistence and their role in developing new therapeutic modalities and compounds to optimize

\section{Dovepress}

clinical outcomes for existing disease states are major areas of interest for the journal. This journal has been accepted for indexing on PubMed Central. The manuscript management system is completely online and includes a very quick and fair peer-review system, which is all easy to use. Visit http://www. dovepress.com/testimonials.php to read real quotes from published authors. 